\title{
Linfoma cutâneo em equino Quarto de Milha
}

\author{
Cutaneous lymphoma in a Quarter Mile horse \\ Fernanda Carlini Cunha dos Santos' ${ }^{1}$ Lays Wouters Ugolini' ${ }^{1}$, Henrique Ramos Oliveira', \\ Tanise Policarpo Machado² \& Leonardo Porto Alves'
}

\begin{abstract}
Background: Lymphoma, although rare, is the most common hematopoietic neoplasia in horses. The overall incidence of lymphoma is between 1.3-2.8\% of all equine neoplasia and it has a prevalence of $0.002-0.5 \%$ in the equine population. Lymphoma can be classified as multicentric, alimentary, mediastinal, cutaneous and solitary. The cutaneous is the rarest form and it usually presents with multifocal skin lesions, with no other clinical signs. The diagnoses is accomplished by histopathological examination of a biopsy or cytological examination of a fine needle aspirate. The aim of the current study is to report a case of the rarest form of equine lymphoma, the cutaneous.

Case: An 8-year-old equine female, Quarter Mile, was evaluated due to volume's increase and subcutaneous nodules disseminated along the body. These lesions developed gradually during 2 years. The mare was used for ridding, it was kept in the field with 10 other equines and was the only one affected. The mare was vaccinated for influenza and was negative for glanders and equine infectious anemia. During clinical exam, all vital parameters were within limits and body condition score was classified as 6 (Henneke Chart). It was observed bilateral nasal secretion and depigmentation in ocular and vulvar mucous. It was observed multiple delimited areas with size ranging from few $\mathrm{cm}$ up to $7 \mathrm{~cm}$, hard, mobile, painless, located in the subcutaneous and disseminated in the body (including head, neck, thorax, limbs and perineum). Red blood cell, leucocytes, fibrinogen, total plasmatic protein were within normal limits. Due to clinical signs and the potential risk of a zoonosis, the glanders test was repeated (complement fixation test) and the result was negative. Differential diagnoses also included insect hypersensitive, which was discarded since the female did not presented pruritus nor alopecia, lesions gradually increased in size and no seasonality was observed. It was performed excisional biopsy for tissue culture, which revealed no growth of aerobic mesophile bacteria. Histopathological evaluation revealed rounded cell proliferation similar to lymphocytes situated in the deep derma and subcutaneous. After evaluation of history, clinical exam and complementary exams the mare was diagnosed with cutaneous lymphoma. The owner was instructed that there was no available specific treatment with good efficacy and viable for equines at this stage. Besides, it is important to evaluated the mare constantly due the possibility of future lesions in organs or intern lymph nodes.

Discussion: Cutaneous lymphoma is an uncommon disease, especially in horses, that can present with variable clinical signs, immunosuppression, rapid systemic disease progression or none at all. In the present case report, during clinical examination lesions were observed in areas of lymphatic drainage. Identification of neoplastic lymphocytes during cytological examination or histopathological evaluation of biopsy tissue can confirm the presence of lymphoma, as performed in the present case. Treatment is palliative and occasionally results in complete cure, mainly in equine with single lesions. The mare had cutaneous lymphoma disseminated all long the body and no clinical signs that could suggest gastrointestinal neoplastic lesions, even though the owner was advised that this animal should be monitored regularly in the future, specially due the possibility of metastatic lesions in any other organ. In equine, lymphoma has low incidence and the cutaneous form is the rarest one. Clinical signs are typically non specific and develop insidiously, so it is important to perform complementary exams for accurate diagnoses and for differential diagnoses of tegumental and infectious diseases.
\end{abstract}

Keywords: lymphadenopathy, lymph nodes, neoplasia, tumor.

Descritores: linfoadenopatia, linfonodos, neoplasia, tumor. 


\section{INTRODUÇÃO}

O linfoma (linfossarcoma ou linfoma maligno) é a neoplasia mais frequente do sistema hematopoiético de equinos, atingindo tecidos linfoides, incluindo linfonodos, baço e cadeias de linfonodos mesentéricos [27]. Em equinos, a incidência de linfoma é de 1,3-2,8\% considerando todas as neoplasias e a prevalência é de 0,002-0,5\% considerando toda população [24,25]. Em um estudo brasileiro, foram detectados 133 (2\%) alterações neoplásicas em 6.669 equídeos, sendo que destas, $4 \%$ (3/133) foram diagnosticadas como linfoma [1].

O linfoma é classificado em multicêntrico (ou generalizado), gastrintestinal, mediastinal, cutâneo e extranodal. O multicêntrico é a forma mais comum, caracterizado pelo envolvimento de múltiplos linfonodos (periféricos e/ou internos) e órgãos (principalmente fígado, baço, medula óssea, intestinos e rins), devido à distribuição de linfócitos neoplásicos pela circulação linfática [17,18,20,21,24,25,32]. O gastrintestinal corresponde a aproximadamente $19 \%$ dos casos de linfoma [28], afetando segmentos intestinais. O mediastinal é o mais comum da cavidade torácica. O cutâneo é a forma menos comum de linfoma, caracterizado por lesões multifocais, nódulos subcutâneos que podem resultar em áreas de alopecia, ulcerações e exsudato amarelado $[2,7,9,12]$. O extranodal inclui o envolvimento único de órgãos, incluindo baço, palato, nasofaringe, língua, meninges e pelve $[3,8,14,15,19,23,29,33]$.

A manifestação clínica de casos de linfoma é inespecífica, variando em função do órgão acometido, grau de comprometimento e tempo de evolução $[25,27]$. Os sinais clínicos mais comuns são anorexia, depressão, perda de peso, febre, linfadenopatia, edema na porção ventral do abdômen e na região distal dos membros [20,25,27].

O objetivo deste estudo foi relatar o caso de um equino apresentando a forma menos comum de linfoma, a cutânea.

\section{CASO}

Foi atendido no Hospital Veterinário da Universidade de Passo Fundo um equino, fêmea, da raça Quarto de Milha, com 8 anos de idade, apresentando nódulos subcutâneos disseminadas pelo corpo. O proprietário relatou que a fêmea desenvolveu estas lesões há aproximadamente dois anos, aumentando de volume gradativamente. Há aproximadamente um ano, a égua apresentou um quadro compatível com obstrução recorrente das vias aéreas, sendo submetida a tratamento e apresentando melhora clínica. A fêmea era utilizada para montaria por crianças dentro da propriedade, permanecia em piquete com mais 10 equinos de montaria, sendo a única afetada. Não havia histórico de transporte de animais nos últimos três anos. A paciente possuía vacinação para influenza e atestado negativo para anemia infecciosa equina e mormo.

No exame físico geral, não foram observadas alterações nos parâmetros vitais e o escore de condição corporal foi classificado como 6 (escala Henneke Chart) [10]. Observou-se discreta secreção nasal serosa e áreas de despigmentação na mucosa ocular e vulvar. Foram observadas múltiplas áreas circunscritas com tamanho de até $7 \mathrm{~cm}$, firmes a palpação, com mobilidade, indolor, localizadas no tecido subcutâneo, disseminadas pelo corpo (incluindo cabeça, pescoço, tórax, membros e períneo - Figuras 1, 2 e 3)

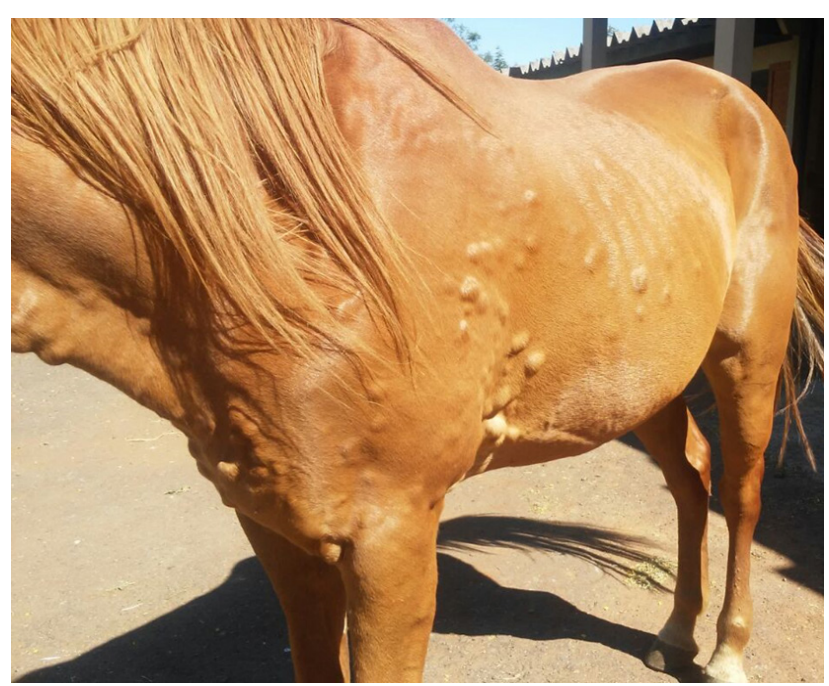

Figura 1. Linfoma cutâneo - nódulos em pescoço e tórax de um equino.

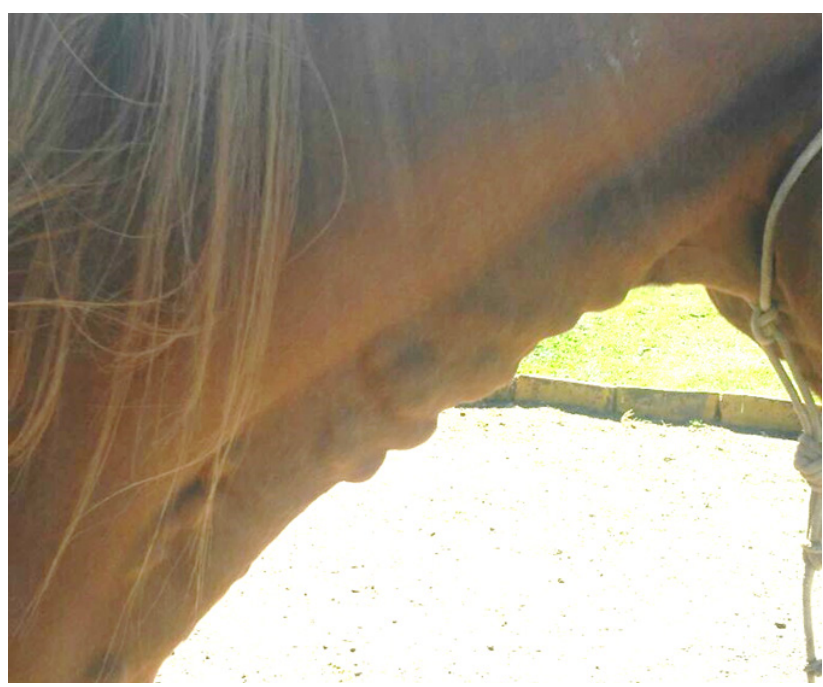

Figura 2. Linfoma cutâneo - nódulos em pescoço de um equino. 


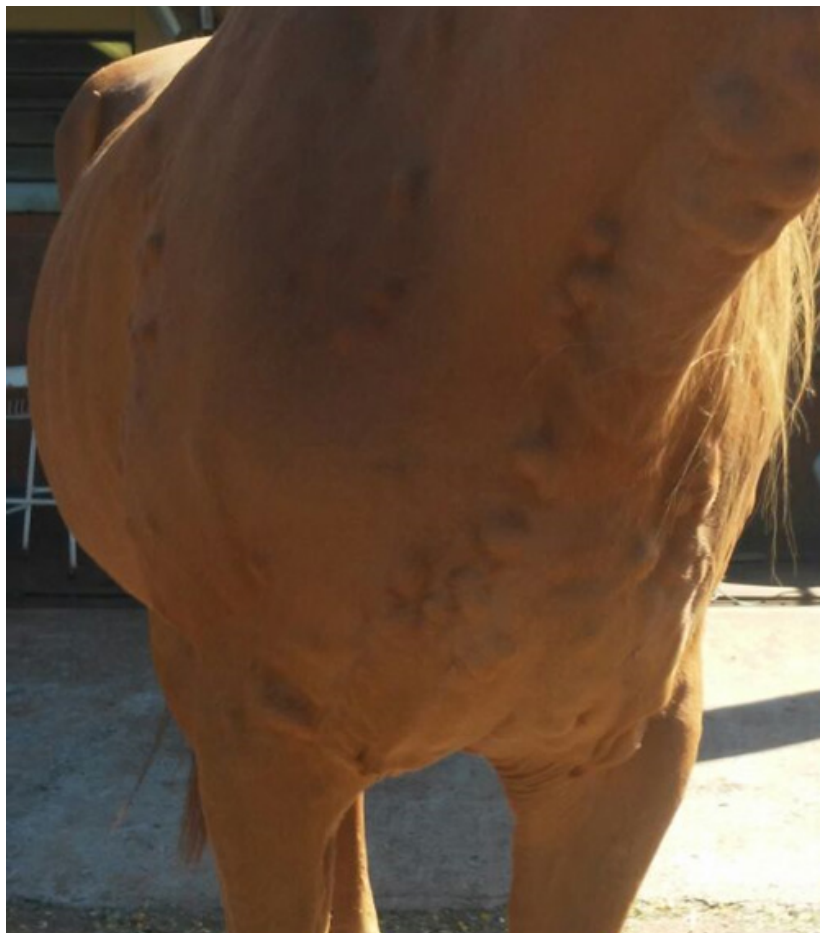

Figura 3. Linfoma cutâneo - nódulos em pescoço, tórax e membros anteriores de um equino.

Foi realizado avaliação do hemograma, leucograma, fibrinogênio e proteínas plasmáticas totais, sem alterações dignas de nota.

O exame de mormo foi repetido (pela técnica de fixação de complemento) devido as características clínicas das lesões e o risco de transmissão, com resultado negativo.

No diagnóstico diferencial foi considerado a hipersensibilidade à picada de insetos, entretanto a fêmea não apresentava prurido e alopecia, com as lesões apresentando aumento gradativo e sem sazonalidade (não houve remissão ao longo dos anos).

Foi realizado biópsia excisional seguida de cultura de tecidos (sem crescimento de bactérias aeróbias mesófilas) e exame histopatológico, sendo observado microscopicamente proliferação de células arredondadas similares a linfócitos localizada em derme profunda e tecido subcutâneo, densamento celular e expansiva (Figuras 4 e 5). Após a avaliação do histórico, exame clínico e exame histopatológico definiu-se o diagnóstico definitivo como linfoma cutâneo.

O proprietário foi instruído que esta doença neoplásica não apresenta tratamento específico, viável e eficaz para equinos neste estágio. Além disso, foi orientado sobre a necessidade de monitoração da paciente devido a probabilidade de desenvolvimento de lesões em órgãos e linfonodos internos.

\section{DISCUSSÃO}

A etiologia do linfoma é desconhecida, nenhum fator de risco foi estabelecido para sua ocorrência, sem predisposição por sexo, raça, pelagem ou idade [25]. A maioria dos casos reportados ocorre em equinos considerados adultos, com idade variando entre $4 \mathrm{e}$ 10 anos [25,27]. Há também relatos em equinos com idade inferior a 1 ano, em feto abortado e em animais idosos $[6,9,24,26,30]$.

No presente relato, durante o exame clínico, foi observado que as lesões estavam localizadas em regiões compatíveis com áreas de drenagem de cadeias linfáticas. Em relação ao diagnóstico diferencial e considerando o histórico (doença cutânea crônica), achados clínicos (corrimento nasal, nódulos subcutâneos, linfoadenopatia), exposição de humanos e outros equídeos, foi realizado novamente o exame para detecção da zoonose mormo. O resultado foi negativo, liberando a égua para contato com outros animais e humanos, no entanto ressalta-se a importância da inclusão

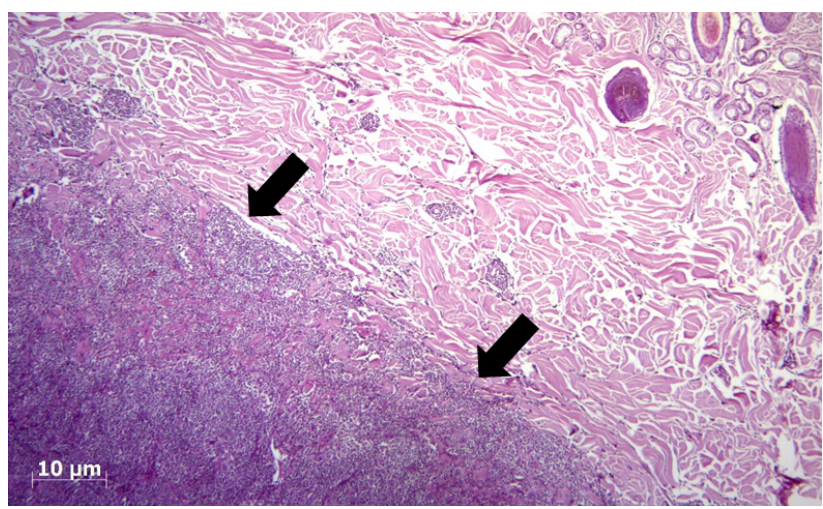

Figura 4. Avaliação histológica de linfoma cutâneo em equino. Extensa massa de linfócitos neoplásicos em derme profunda (indicado por setas pretas), estendendo-se ao subcutâneo [Hematoxilina e eosina, 10X].

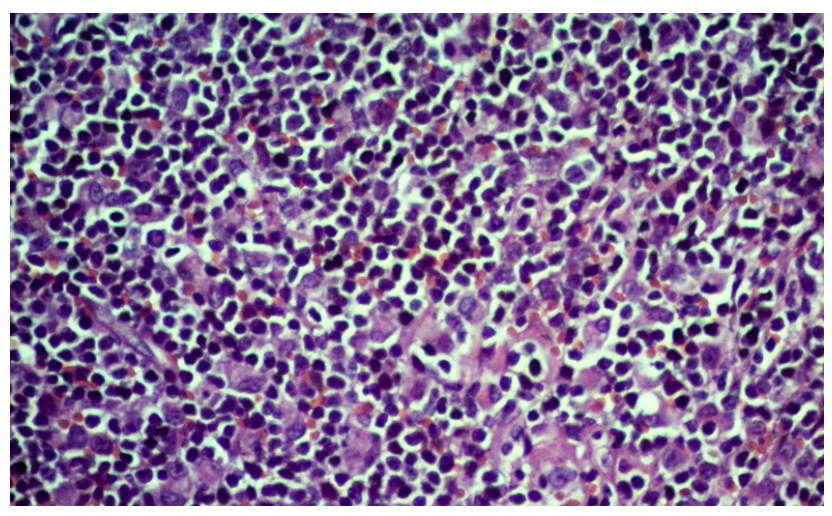

Figura 5. Avaliação histológica de linfoma cutâneo em equino. Marcada proliferação de pequenos linfócitos atípicos com estreita relação núcleo citoplasma e núcleo hipercromático, além de infiltrado de macrófagos e plasmócitos [Hematoxilina e eosina, 40X]. 
deste exame no diagnóstico diferencial conforme a apresentação do caso.

O diagnóstico definitivo do presente relato foi baseado nos achados histopatológicos, permitindo a avaliação da arquitetura do órgão coletado (cadeia linfática) e detecção infiltração neoplásica, conforme descrito em literatura [27].

O exame histopatológico é considerado o método de eleição para diagnóstico definitivo de linfoma, além disso, pode ser realizado exame de imunohistoquímica e imunofenotipagem. A imunohistoquímica é uma técnica que inclui a utilização de anticorpos como reagentes específicos para determinação do subtipo de linfoma e determinação da taxa de proliferação celular. A imunofenotipagem é o método preferencial para a determinação da linhagem celular e análise da maturação das células nas neoplasias hematológicas [27]. No entanto, como principal desvantagem, temos que o desenvolvimento de um sistema de classificação para o linfoma em equinos é dificultado pela falta de marcadores específicos para a espécie [13].

Para estabelecimento da melhor abordagem terapêutico do linfoma, previamente deve ser avaliado o estado geral de saúde do paciente, extensão, localização da lesão, disponibilidade de investimento e comprometimento do proprietário [4,31]. Além disso, na maioria dos casos, o tratamento é somente paliativo, não resultando em cura.

A radioterapia apresenta alto custo e necessita de equipamentos específicos, atualmente indisponíveis na maioria dos hospitais veterinários brasileiros, além disso, a resposta ao tratamento varia conforme o tamanho e localização do linfoma. Os princípios da quimioterapia são maximizar a morte de células neoplásicas e reduzir local e sistemicamente os efeitos colaterais [27]. A maioria dos protocolos inclui terapia multimodal por longo período resultando em remissão do linfoma, no entanto, uma vez descontinuada a terapia, as lesões retornam [4].

A remoção cirúrgica é indicada nos casos de linfomas solitários e/ou em pouca quantidade, já tendo sido realizada em cólon maior, globo ocular e trato respiratório anterior [3,5,22]. Neste caso clínico, a remoção cirúrgica seria impraticável, considerando que a égua apresentava múltiplas lesões, com tamanhos variáveis e em diferentes regiões do corpo.

O sistema gastrintestinal pode ser afetado pelo linfoma como doença neoplásica primária (caracterizando o linfoma alimentar), representar parte de uma alteração generalizada (caracterizando o multicêntrico) ou ser resultante de uma disseminação metastática a partir de outro foco primário em qualquer órgão ou tecido [16]. No presente relato, o equino não apresentou sinais clínicos que sugerissem acometimento por linfoma em outras regiões, exceto os nódulos cutâneos. Todavia, o proprietário foi informado acerca da necessidade de acompanhamento futuro do animal quanto a evolução da neoplasia e possível surgimento de metástases.

Em equinos, o linfoma tem baixa incidência e a forma cutânea é a menos comum. No entanto, devido à natureza insidiosa da neoplasia e a ausência de sinais clínicos patognomônicos, esta doença neoplásica deve ser incluída no diagnóstico diferencial de doenças cutâneas e infectocontagiosas. O diagnóstico definitivo foi baseado no histórico e avaliação clínica, sendo confirmado pelo exame histopatológico.

Declaration of interest. The authors report no conflicts of interest. The authors alone are responsible for the content and writing of the paper.

\section{REFERENCES}

1 Baccarin R.Y.A., Ida K.K., Oliveira S.L.P., Matushima E.R., Belli C.B. \& Fernandes W.R. 2011. Ocorrência de neoplasia em 15 anos de atendimento hospitalar de equídeos. Brazilian Journal of Veterinary Research and Animal Science. 48(6): 439-445.

2 Bruijn C.M., Veenman J.N., Rutten V.P., Teske E., van Nieuwstadt R.A. \& Ingh T.S. 2007. Clinical, histopathological and immunophenotypical findings in five horses with cutaneous malignant lymphoma. Research in Veterinary Science. 83(1): 63-72.

3 Burba D., Jann H. \& Confer A. 1991. Surgical reduction of a laryngeal lymphosarcoma mass causing dyspnea in a horse. Equine Practice. 13(3): 14-18.

4 Burns T. \& Couto C. 2009. Systemic chemotherapy for oncologic diseases. In: Robinson N \& Sprayberry K. (Eds). Current Therapy in Equine Medicine. 6th edn. St. Louis: Saunders, pp.15-18.

5 Dabareiner R.M., Sullins K.E. \& Goodrich L.R. 1996. Large colon resection for treatment of lymphosarcoma in two horses. Journal of American Veterinary Medical Association. 208(6): 895-897.

6 Dewes H.F. \& Blakeley J.A. 1980. Lymphosarcoma in a thoroughbred filly. New Zealand veterinary Journal. 28(4): 82. 
7 Epstein V. \& Hodge D. 2005. Cutaneous lymphosarcoma in a stallion. Australian Veterinary Journal. 83(10): 609-611.

8 Gerard M.P., Healy L.N., Bowman K.F. \& Miller R.T. 1998. Cutaneous lymphoma with extensive periarticular involvement in a horse. Journal of American Veterinary Medical Association. 213(3): 391-393.

9 Haley P.J. \& Spraker T. 1983. Lymphosarcoma in an aborted equine fetus. Veterinary Pathology. 20(5): 647-649.

10 Henneke D.R., Potter G.D., Kreider J.L. \& Yeates B.F. 1983. Relationship between condition score, physical measurements and body fat percentage in mares. Equine Veterinary Journal. 15(4): 371-372.

11 Jacobs R., Messick J. \& Valli V. 2002. Tumors of the skin and hemolymphatic system. In: Meuten D. (Ed). Tumors of Domestic Animals. 4th edn. Ames: Iowa State Press, pp.119-198.

12 Johnson P.J. 1998. Dermatologic tumors (excluding sarcoids). Veterinary Clinics of North America: Equine Practice. 14(3): 625-658.

13 Kelley L.C. \& Mahaffey E.A. 1998. Equine malignant lymphomas: morphologic and immunohistochemical classification. Veterinary Pathology. 35(4): 241-252.

14 Lane J.G. 1985. Palatine lymphosarcoma in two horses. Equine Veterinary Journal. 17(6): 465-467.

15 Lester G.D., MacKay R.J. \& Smith-Meyer B. 1992. Primary meningeal lymphoma in a horse. Journal of American Veterinary Medical Association. 201(8): 1219-1221.

16 Mair T.S., Yeo S.P. \& Lucke V.M. 1990. Hypercalcaemia and soft tissue mineralisation associated with lymphosarcoma in two horses. Veterinary Record. 126(5): 99-101.

17 McClure J.T. 2000. Leukoproliferative disorders in horses. Veterinary Clinics of North American Equine Practitioner. 16(1): 165-182.

18 Meyer J., Delay J. \& Bienzle D. 2006. Clinical, laboratory, and histopathologic features of equine lymphoma. Veterinary Pathology. 43: 914-924.

19 Montgomery J.B., Duckett W.M. \& Bourque A.C. 2009. Pelvic lymphoma as a cause of urethral compression in a mare. Canadian Veterinary Journal. 50(7): 751-754.

20 Munoz A., Riber C., Trigo P. \& Castejon F. 2009. Hematopoietic neoplasias in horses: Myeloproliferative and lymphoproliferative disorders. Journal of Equine Science. 20(4): 59-72.

21 Neufeld J.L. 1973. Lymphosarcoma in the horse: a review. Canadian Veterinary Journal 14(6): 129-135.

22 Rebhun W.C. \& Del Piero F. 1998. Ocular lesions in horses with lymphosarcoma: 21 cases (1977-1997). Journal of American Veterinary Medical Association. 212(6): 852-854.

23 Rhind S.M. \& Dixon P.M. 1999. T cell-rich B cell lymphosarcoma in the tongue of a horse. Veterinary Record. 145(6): 554-555.

24 Savage C.J. 1998. Lymphoproliferative and myeloproliferative disorders. Veterinary Clinics of North America: Equine Practice. 14(3): 563-578.

25 Schneider D. 2003. Lymphoproliferative and myeloproliferative disorders. In: Robison N. (Ed). Current Therapy in Equine Medicine. 5th edn. St. Louis: Saunders, pp.359-362.

26Seahorn T., Carter G., Morris E., Lay J. \& Ludwig K. 1988. Lymphosarcoma in a foal: a case report. Journal of Equine Veterinary Science. 8(4): 317-319.

27 Taintor J. \& Schleis S. 2011. Equine Lymphoma. Equine Veterinary Education. 23(4): 205-213.

28 Taylor S.D., Pusterla N., Vaughan B., Whitcomb M.B. \& Wilson W.D. 2006. Intestinal neoplasia in horses. Journal of Veterinary Internal Medicine. 20(6): 1429-1436.

29 Tanimoto T., Yamasaki S. \& Ohtsuki Y. 1994. Primary splenic lymphoma in a horse. Journal of Veterinary Medical Science. 56(4): 767-769.

30 Tomlinson M.J., Doster A.R. \& Wright E.R. 1979. Lymphosarcoma with virus-like particles in a neonatal foal. Veterinary Pathology. 16(5): 629-631.

31 Vail D. \& Young K. 2002. Hematopoietic tumors. In: Withrow S. \& Vail D. (Eds). Small Animal Clinical Oncology. 4th edn. St. Louis: Saunders, pp.699-784.

32 van den Hoven R. \& Franken P. 1983. Clinical aspects of lymphosarcoma in the horse: a clinical report of 16 cases. Equine Veterinary Journal. 15(1): 49-53.

33 Weaver M., Dobson J. \& Lane J. 1996. Treatment of intranasal lymphoma in a horse by radiotherapy. Equine Veterinary Journal. 28(3): 245-248. 